\title{
Mobile Support for Adults with Mild Learning Disabilities during Clinical Consultations
}

\author{
Ryan Colin Gibson \\ Dept. of Computer and \\ Information Sciences, \\ University of Strathclyde, \\ Glasgow, \\ ryan.gibson@strath.ac.uk
}

\author{
Dr. Matt-Mouley Bouamrane \\ Dept. of Computer and \\ Information Sciences, \\ University of Strathclyde, \\ Glasgow, \\ mattmouley.bouamrane@strath.ac.uk
}

\author{
Dr. Mark Dunlop \\ Dept. of Computer and \\ Information Sciences, \\ University of Strathclyde, \\ Glasgow, \\ mark.dunlop@strath.ac.uk
}

\begin{abstract}
Previous studies have suggested that the learning disability (LD) population face significant communication barriers when interacting with health professionals. Such obstacles may be considered as preventable; however, there is a surprising lack of research-based technologies available that intend to promote this communication. We aim to address this issue by investigating the potential use of mobile technologies to support adults with mild LDs during clinical consultations. To achieve this, we interviewed 10 domain experts including government advisors, academics, support workers and General Practitioners. The extracted information was used to develop an initial technology probe, which was evaluated by a subset of the aforementioned experts. The overall contribution of this research is a set of design guidelines for the development of Augmentative and Communicative technologies that target the clinical needs of adults with mild LDs.
\end{abstract}

\section{Author Keywords}

Learning disabilities; clinical consultations; AAC technologies

\section{ACM Classification Keywords}

H.1.2 User/Machine Systems: Human Factors. K.4.2 Social Issues: Assistive technologies for persons with disabilities.

\section{INTRODUCTION}

People with learning disabilities (LDs) often have greater health needs than the general population [30]. This may in part contribute to the significant reduction in the life expectancy of the LD community, with men dying on average 13 years younger in the United Kingdom [22]. This figure rises to 20 for females and is a trend that may be

Permission to make digital or hard copies of all or part of this work for personal or classroom use is granted without fee provided that copies are not made or distributed for profit or commercial advantage and that copies bear this notice and the full citation on the first page. Copyrights for components of this work owned by others than ACM must be honored. Abstracting with credit is permitted. To copy otherwise, or republish, to post on servers or to redistribute to lists, requires prior specific permission and/or a fee. Request permissions from Permissions@acm.org.

MobileHCI '18, September 3-6, 2018, Barcelona, Spain

(C) 2018 Association for Computing Machinery.

ACM ISBN 978-1-4503-5898-9/18/09 ...\$15.00

https://doi.org/10.1145/3229434.3229469 recognized throughout the developed world [21]. However, genetics cannot be held solely responsible for such disparity. For example, an enquiry into the premature deaths of people with learning disabilities concluded that approximately half of all deaths studied were potentially avoidable, with $27.5 \%$ of these amenable to better quality health care [16]. This suggests that people with learning disabilities are subjected to various health inequalities. Previous literature has supported this theory and inferred that many of these barriers are potentially avoidable including: issues accessing healthcare services; undereducated staff; care-givers acting as intermediaries; inappropriate/inflexible processes or procedures; and insufficient collation or use of healthcare data $[1,19,30]$.

In this context the term "learning disability" may be applied to an individual if they satisfy the following 3 criteria: the person's intellectual functioning is impaired; the person's adaptive/social functioning is impaired; and these two conditions occur before adulthood [34]. There are several different types of learning disabilities; however, those with mild LDs are generally able to communicate their needs but struggle to understand complex concepts. As such, their ability to communicate about medical conditions is significantly affected - a skill deemed crucial to the success of consultations [17]. Further potential impairments that contribute to this include: reduced receptive and expressive skills; a restricted knowledge of the human body meaning they may fail to recognize or describe potential symptoms accurately; and limitations in their abstract thinking and long term which may affect their ability to provide an accurate medical history $[5,19,27,33]$. To accommodate for these deficiencies, patients may make use of Alternative and Augmentative Communication (AAC) devices. But such technologies tend to be developed to support everyday communication and may lack the features required to address the needs of patients with LDs in a clinical environment.

Work in related areas such as rehabilitation and remote consultation has shown great promise and support for people with other special needs $[2,8,11]$. Throughout this paper, we intend to demonstrate how mobile AAC technologies may be used to address the complex needs of 
adults with mild learning disabilities in the medical domain. To achieve this, we have conducted a series of semistructured interviews involving a purposive selection of experts in learning disabilities including: 2 General Practitioners; 3 governmental advisors who were involved in the development of Scotland's national learning disability strategy; 4 academics in the fields of social work, cognitive psychology, inclusive education, and aging, fragility and dementia; and a full-time support worker. The interviews contributed to the design of a technology probe that was subsequently presented to a subset of the experts for evaluation. The information obtained from these studies has resulted in a set of design guidelines for the development of future clinical AAC technologies for people who have mild learning disabilities.

We expect such technologies to have a significant impact in the future of clinical consultations. Many General Practitioners (GPs) feel ill equipped when attempting to overcome the aforementioned communication barriers [27] and are unable to find the time to update their knowledge of learning disabilities to achieve this [23]. AAC technologies have the potential to convey information in a format understood by both sets of stakeholders and may be learned in a short period of time. This may improve the success of consultations by increasing the depth and accuracy of information extracted from patients with mild LDs.

\section{CONTRIBUTIONS}

The main contributions of this paper are as follows:

- An initial set of requirements for clinical AAC technologies that target the needs of adults with mild learning disabilities.

- Using these requirements, we designed a technology probe that aims to explore how tablet technologies may be used to support adults with mild LDs during clinical consultations.

- Through a series of evaluation studies, we demonstrated the potential impact AAC technologies may have on consultations involving patients with mild LDs.

\section{RELATED WORK}

A number of studies in the fields of health and Human Computer Interaction have had a significant influence on our research. Firstly, Heslop et al. and academics at Lancaster University $[16,20]$ have identified a number of health conditions that are prevalent throughout the LD community, including those that are frequently overshadowed by medical professionals. These conditions include: certain types of cancer such as gastrointestinal cancers; epiliepsy; cardiovascular disease; diabetes; and constipation amongst others. AAC technologies may target such ailments in depth to ensure they are brought to the attention of medical professionals and are treated appropriately.

Various studies have been conducted that have reviewed the effectiveness of current AAC technologies [3, 25, 29].
Baxter et al. found several issues to be common throughout a range of traditional AAC technologies including: operational difficulties and time; complexity; limited flexibility; self-confidence; and cost [3]. Furthermore, continuous access to support was crucial to the success of such technologies. McNaughton \& Light and Niemeijer et al. $[25,29]$ concluded that AAC applications embedded within everyday mobile technologies have the potential to mitigate some of these problems. However, they introduce issues of their own including: small screen size; and the potential of being introduced without considering the specific needs of the user.

Current communication aids embedded within the clinical domain focus on tailoring the care process to suit the individual needs of the patient. For example, Bell \& Cameron [4] utilized the Talking Mats ${ }^{\mathrm{TM}}$ framework to asses the psychological status of a woman with mild learning disabilities. The images presented to the patient were designed to depict the key components of her life and enabled her to discuss each aspect freely and confidently which led to an accurate diagnosis being carried out. Furthermore, hospital passports, such as the one described by Brodrick et al. [7], are slowly becoming established throughout secondary care in the United Kingdom. The passports capture essential personal information from patients about their care needs (communication habits, environmental or medical requirements etc.), and this ensures that staff interact with the patient in a consistent manner and accommodate for their individual demands.

However, the research most closely related to our own comes in the form of an online questionnaire that supports children in reporting their psychological health [3]. Many of the conclusions made were considered relevant within the design of our interface, including: the need to present one question at a time; embedding a limited amount of information within each page; and using a range of modalities to convey this information, amongst others. Initial results from the study (project is still ongoing) were promising, with a range of users being able to reliably complete the questionnaire. This highlights the potential success such technologies may have in extracting accurate medical information from people who have LDs.

\section{Methodology}

The research conducted within this paper is part of a wider project that aims to develop (in conjunction with the views of target stakeholders) a digital aid that supports adults with mild learning disabilities during the consultation process. To ensure the intervention is developed in a structured manner, the authors are following MRC's framework for Complex Interventions [10]. The overall process may be found in Figure 1 and is highly appropriate for this research since it emphasizes the collection of evidence at various points throughout a project. This ensures that the intervention is effective in achieving its goal and presents multiple opportunities to reevaluate the design if this is not the case. 


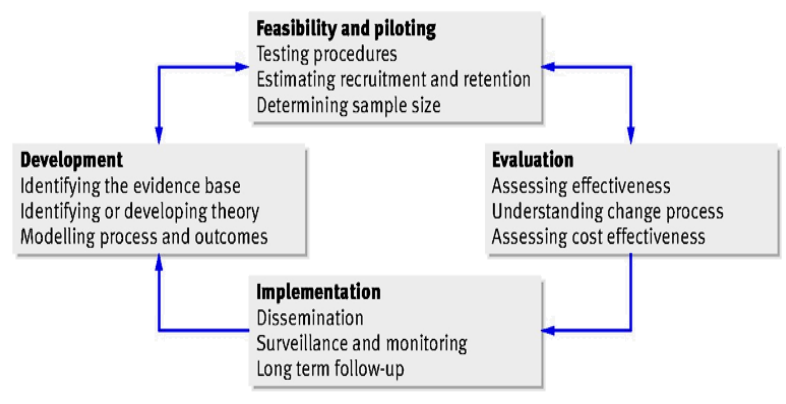

Figure 1: MRC Framework for Complex Interventions [10]

The authors are currently focusing on the "Development" phase. We conducted a separate scoping review that aimed to explore the barriers to accessing effective healthcare for adults with mild LDs, and the current technologies used to overcome these. This provided an evidence base for the proposed intervention, since no high-tech AAC technologies had been identified that addressed the needs of the target population within the medical domain.

Nonetheless, the literature discussed a wide range of strategies that have had some success in promoting everyday communication and these formed part of the requirements for the proposed aid. The lead author then proceeded to conduct a series of semi-structured interviews with the aforementioned experts to validate these findings and to extract further requirements for the application. Norman suggests that stakeholders are initially unaware of their requirements for a system during its early design stages [31] and may benefit from interacting with similar technologies to clarify their views. Therefore, the decision was made to involve experts within a round of interviews in order to develop and subsequently present a technology probe to target stakeholders during future requirements gathering studies. Although a co-design process would have resulted in a more innovative solution, we believe our interface highlights several features that developers must be made aware of during the future creation of medical AAC technologies. The target number of experts to be interviewed was set at between 10 and 15 people to ensure a wide range of knowledge was utilized during the initial design process. The average duration of the interviews was approximately 34 minutes.

All participants were then invited to participate within a series of usability tests, with 4 giving their consent (experts $1,2,4 \& 8)$. The purpose of these tests was to inform the requirements extracted previously. The experts were required to complete a series of tasks using the probe, and then comment on the appropriateness of the intervention for the target population. The sample size was set at between three and five participants as Dumas and Redice suggested that such a population enables key design and usability flaws to be identified and subsequently addressed over a short period of time [12]. Further investigations may then be carried out where required. The tasks took approximately 21 minutes to complete and the results obtained were used to update the requirements listed in the next section. No assistance was provided during the completion of the scenarios to ensure key design flaws were naturally identified.

All studies were conducted under institutional ethical approval and a framework analysis [13] of the transcribed interviews was carried out to produce a structured summary of the views held by the participants. Excerpts from these summaries were then used to shape the design of the probe described in this paper and the resulting tables have been made available at: http://dx.doi.org/10.15129/7fed3a659ac4-4152-953b-b606376b64b5. The IDs' listed throughout refer to the expert's position in the requirements gathering framework analysis table.

\section{REQUIREMENTS}

A plethora of potential requirements were discussed throughout the interview process, due to the range of expertise held by the participants. Consequently, it was important to discern which of these apply to the majority of stakeholders and those that cater for more individual needs. The authors achieved this by implementing those requirements that occurred as common themes across the interviews conducted. Traits disclosed by individual participants were embedded within the application providing they had been supported by previous literature. We believe that this process has resulted in the creation of a prototype that addresses the common needs of adults with mild learning disabilities and may therefore be utilized by a range of stakeholders. The requirements implemented may be found in table 1

\section{Requirement Description}

[1] Information should be conveyed using a range of modalities including: text, immediately identifiable images, and speech.

[2] A minimum font size of 14 should be used with buttons and text being made as large as possible.

[3] Images and text used to represent potential symptoms should be developed in conjunction with the views of target stakeholders. Medical jargon should be avoided where appropriate.

[4] The consultation process should be broken down into manageable chunks by presenting small, closed questions that focus on solitary ideas.

[5] The number of options presented to the user should be restricted to a maximum of 4 .

[6] The aesthetics of the aid should be made customizable to cater for individual needs.

[7] Questions should focus on the specific health needs of target stakeholders
ID

$1,3-5$, $7-10$

$3-5,8-$

10

$2,3,5$,

8,10

$3-4,9-$

10

3-4, 9-

10

$4-5,8$, 10

$1-2,10$ 


\section{Requirement Description}

[8] The number of clicks required to complete the process should be reduced to a minimum

[9] A consistent layout should be provided including the option to access a help feature at all times.

[10] Questions should aim to extract the symptoms experienced by patients, the duration and history of these symptoms, and the overall health of patients.

[11] A record should be kept of all the key activities made by a patient.

[12] The aid should be portable.

\section{Table 1: Main requirements extracted from the experts interviewed}

The requirements have been ordered to depict the number of experts who had proposed them throughout the interviews. Those requirements that had been referenced the same number of times were then ordered to reflect the depth in which they were discussed by the participants. It is important to note that some of the listed requirements do not specifically cater for the needs of people with LDs, but instead cater for additional manifestations that occur frequently within the learning disability population. For example, many target stakeholders will have significant visual deficiencies and may therefore require larger font sizes to read text. Others may have significant motor deficiencies meaning clickable objects have to be increased in size and spaced far apart. Illiteracy is also a common issue meaning other modalities must be used to convey information. Accommodating for these limitations may result in the needs of other vulnerable populations being catered for such as the elderly.

\section{DESIGN}

\section{Adaptive Questionnaire}

Requirement 7 states that the number of clicks needed to complete the application's process should be reduced to a minimum. Therefore, we have limited the amount of irrelevant questions presented by implementing a dynamic stack-based questionnaire similar to that proposed by Bouamrane et al. [6]. In short, a main questionnaire stack is created according to the primary symptom selected by the patient. This stack consists of the questions vital to extracting the cause of the patient's condition. The questions are popped sequentially from the top and presented to the user providing they uphold certain preconditions e.g. symptoms relating to pregnancy will only be forwarded to those who are female. The symptoms selected may then result in additional questions being pushed to the top of stack for further exploration. For example, the questions designed to extract the symptoms of blepharitis may only be added if the user has indicated they have itchy red eyes. This strategy therefore restricts the number of questions presented to those deemed relevant to the user's condition and may have significant advantages for adults who have short attention or working memory spans. The cognitive load placed on patients with LDs is also reduced.

\section{User Interface}

Adults with mild learning disabilities are heterogeneous in nature and may not respond to information in a similar manner. For example, some may be unable to participate within verbal conversations due to the complexity of the language being used [32]. Consequently, the experts interviewed suggested that a range of communication modalities be targeted when representing potential symptoms including text, immediately identifiable images, and speech.

As shown in figure 2, we have embedded the option to playback any passage of text displayed on screen. This was achieved in the following two ways: firstly, the user may select an audio button (represented by a speaker symbol) located near the passage they wish to play back. This enables patients to have specific sentences read out at will until its meaning is fully understood. However, not all patients possess the cognitive abilities required to read text. As a result, the probe offers these stakeholders the option to playback all passages contained throughout. On completion of page loads, each sentence will be read out sequentially and simultaneously highlighted to ensure that it is clear to the user which passage is being played back. This may also be beneficial to those users who have significant visual impairments.

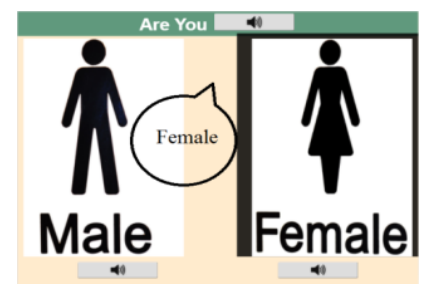

Figure 2: Automatic playback of text.

All options have also been conveyed via the use of images, to accommodate for those stakeholders who are unable to understand the language used. Pictures have been proven to offer an alternative means of depicting language, providing they immediately represent the concept being captured [28]. By targeting these 3 modalities, an increase in comprehension may be obtained as each individual may make use of the communication method that makes most sense to them for each option presented.

As shown in figure 3, the probe requires the user to state whether they are in pain. This further restricts the number of irrelevant questions being presented, since many of the conditions that affect adults with LDs $[16,20]$ may be placed exclusively into one of these categories, and subsequently excluded based on the scale of pain experienced by the patient. We have used drawings similar to those embedded in the Wong Baker smiley faces pain 

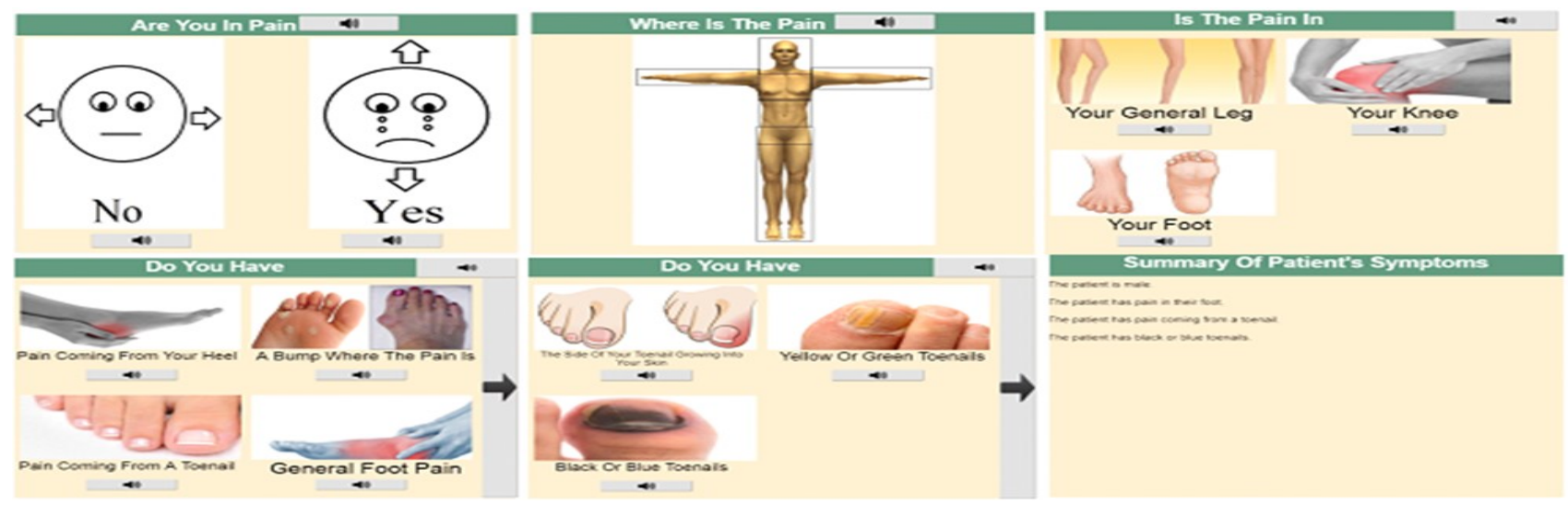

Figure 3: Sample screens representing the selection of symptoms for a bruised toenail.

tool to convey the two distinct emotions. Such a tool has previously enabled people with learning disabilities to successfully report their experience of pain [15]. The use of color was avoided, since participant 10 discussed several scenarios where interviewees focused on the color used and not the emotion conveyed by the image.

The conditions included within the pain/non-pain categories may have an abundance of symptoms that can conceivably affect patients. Rather than displaying all of these at once, the prototype restricts the number of options available to a maximum of 4 related conditions. According to the experts interviewed, adults with mild learning disabilities are frequently omitted from the decision-making process and may struggle to cope with choice. Thus, limiting the amount available may ease the cognitive load placed on stakeholders and may ultimately result in the extraction of more accurate information. As a by-product, the amount of space assigned to clickable objects and to text can be increased and this has significant advantages to those stakeholders who have visual or motor impairments [18]. Reducing the number of symptoms displayed on screen will increase the number of clicks required to complete the process as additional questions will have to be presented. However, the added benefits for those users who have significant cognitive, motor and visual deficiencies warrant the need for these additional questions.

Several of the experts discussed the effectiveness of embedding concrete objects in which the target population may point to. One such object disclosed was the human body. Consequently, patients are required to tap on an image of the body when pinpointing an area causing them distress. This process relies on the user possessing the motor abilities required to tap on small sections of the screen, for example when selecting that they have a pain in their hand. However, many adults with learning disabilities have poor fine motor skills; therefore, the application requires the user to confirm the specific section they have selected. All body parts situated in the proximity of the tap will be presented for the user to triangulate their choice. This also enables those that were unavailable for selection in the original image to be presented.
Since text cannot be relied upon to convey information, key navigational points have been represented via the use of images. This strategy has been proven effective within previous literature, for example in Medhi et als. study that aimed to explore interfaces for illiterate and semi-literate users [26]. We embedded an arrow within the skip button shown in figure 3 , to naturally represent the ability to move on to the next question/page. Ultimately the image used was not clear enough to depict the buttons functionality and the reasons why will be discussed further in the next section. Additional design decisions centered on spacing clickable elements far apart to limit the number of erroneous taps being made; utilizing the limited space available in tablet technologies; and breaking the consultation process down by presenting small, closed questions.

The experts also discussed the need to ensure the intervention is customizable. We agreed with this view to an extent and suggest that the aesthetics of such technologies should be made adjustable. However, since the ordering of questions may be crucial to extracting certain conditions, changes should not be made without approval. Both the language and images used to convey symptoms should be developed in conjunction with the views of target stakeholders and should therefore remain the same within technologies operated by a variety of users.

Each of the requirements listed in this paper have been developed with tablet technologies in mind. Consequently, changes will have to be made when developing similar applications on other devices such as smartphones. For example, due to limited screen space a maximum of 4 options may not be achievable as clickable elements will have to be reduced in size and this may have serious complications for people with motor deficiencies.

\section{USER EVALUATIONS}

Three significant improvements to the design described in the previous section were suggested by the experts involved in the usability studies. Firstly, participant 4 suggested that an accessible summary page of the symptoms selected should be provided for patients as well as practitioners. Such a page should make use of the various communication modalities discussed previously, as shown in the left picture 
of figure 4. This may enable adults with mild learning disabilities to use the information as a visual prompt when discussing their symptoms with a GP. They may also practice the information they wish to convey out with the medical environment.

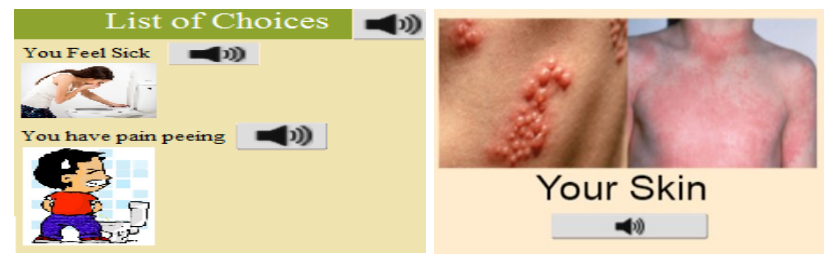

Figure 4: An accessible summary page and image used to depict skin conditions

Both the language and images used within the probe may be considered as placeholders. Currently, there is a lack of guidelines available which discuss how to develop appropriate medical imagery for the LD population. We intend to address this during future co-design workshops involving adults with mild learning disabilities. Nonetheless, the experts were able to identify some flaws in the placeholders used that should be avoided in future studies. Some of the participants believed certain pictures could be taken too literally by the target population. For example, patients may fail to select the picture representing skin conditions (right image of figure 4) if they are suffering from other skin diseases such as eczema. Rather than embedding a range of potential symptoms within this object, it would have been more appropriate to display a general image of the skin.

All of the experts interviewed required an intervention to explain the functionality of the skip button described previously. Recommendations to improve the button therefore focused on ensuring its intention is made clear and included: implementing a help feature across all pages to enable patients to obtain advice when unsure on how to progress; and extending the automatic playback feature to also highlight the button and read out the words "Press here for more options" - although this solution would only assist those who rely on speech. The experts also emphasized the need to embed a return function within the interface to accommodate for mistakes being made.

\section{DISCUSSION}

The experts interviewed were optimistic about the potential impact such technologies may have on clinical consultations. Participant 5 revealed that the funding available for support workers is being cut drastically, and this may result in an increased number of patients attending consultations unaccompanied. Consequently, these stakeholders will require additional support to convey their needs to medical professionals. Participant 8 believes that the current probe has the potential to achieve this since it may be used autonomously by a range of patients. However, by including the target population within the design of the aid and ensuring the technology is tested with a variety of patients, this rate of autonomy may be increased. We intend to achieve this by conducting a round of co-design sessions involving adults with mild learning disabilities and by presenting the existing probe to those participants who are unaware of their needs. The results obtained will be used to update the requirements listed in table 2 before an improved version of the intervention is embedded within the target environment.

Overall, the GPs interviewed stated that they had not used communication aids during previous consultations but were open to the possibility providing the benefits are made clear. This is encouraging considering the success of such technologies relies heavily on the willingness of medical professional to support their use. GPs tend to be undereducated on both the health and communication needs of patients with learning disabilities [1, 19] and this creates significant barriers from the beginning of the consultation. Our probe intends to overcome this by extracting symptoms from the patient out with the appointment. The information may then be used to help shape the questions presented thus enabling practitioners to focus on areas of interest for longer. Furthermore, it has the potential to bring frequently overshadowed conditions to the attention of the GP thus increasing the health of patients. The idea is not to diagnose the patient but instead but instead supply information that may support them in formulating a diagnosis. The information captured may also be used as a referent throughout in order to promote communication. However, participant 6 revealed that practitioners may be skeptical to use data extracted by an algorithm, meaning further GPs should be approached to confirm this view.

\section{CONCLUSION}

To the best of our knowledge, there has been no research conducted into the design of mobile AAC technologies that support the needs of adults with mild LDs in the clinical domain. We have addressed this gap by exploring the feasibility of tablet technologies in supporting these needs. The extracted requirements matched several of the findings made by Bostrom and Eriksson [5] including: the need to present one question at a time; embedding a limited amount of information within each page; and using a range of modalities to convey this information. Furthermore, a number of novel requirements were embedded within the design of the probe including: limiting the questions presented to those deemed relevant to the patient's condition; an automatic audio playback feature; confirming selections that require fine motor skills; and providing an accessible summary of the options selected. Overall, the experts involved throughout the study were optimistic about the potential impact such technologies may have on the consultation process. Opportunities for future work include: investigating the modalities required to represent medical information in a form understood by patients with mild LDs; the development of an ontology that captures the common conditions experienced by the target population; and the co-design of a novel interface based upon the requirements listed in this paper. 


\section{REFERENCES}

1. Alborz, A., McNally, R., Glendinning, C. 2005. Access to health care for people with learning disabilities in the UK: mapping the issues and reviewing the evidence. Journal of Health Services Research \& Policy 10, 173-182

2. Aggarwal, D. Supporting Bodily Communication in Video-based Clinical Consultations. 2016. In: CHI EA '16 Proceedings of the 2016 CHI Conference Extended Abstracts on Human Factors in Computing Systems, 188-192

3. Baxter, S., Enderby, P., Evan, P., Judge, S. 2012. Barriers and Facilitators to the use of High-Technology Augmentative and Alternative Communication Devices: A Systematic Review and Qualitative Synthesis. International Journal of Language \& Communications Disorders 47, 2, 115-129

4. Bell D.M., Cameron L. 2007. From Dare I say...? to I dare say: a case example illustrating the extension of the use of Talking Mats to people with learning disabilities who are able to speak well but unwilling to do so. British Journal of Learning Disabilities 36, 2, 122-127

5. Bostrom, P., Eriksson, E. 2015. Design for SelfReporting Health in Children with Intellectual Disabilities. In: IDC'15 Proceeedings of the 14th International Conference of Interaction Design and Children 279-282

6. Bouamrane, M.M., Rector, A., Hurrell M. 2008. Ontology-Driven Adaptive Medical Information Collection System. In International Symposium on Methodologies for Intelligent Systems Matwin S., Raś Z.W., Ślęzak D. (eds) Foundations of Intelligent Systems. ISMIS 2008. Lecture Notes in Computer Science, vol 4994. Springer, Berlin, Heidelberg pages 574-584

7. Brodrick, D., Lewis, D., Worth, A., Marland, A. 2011. One-page patient passport for people with learning disabilities. Nursing Standard 25, 47, 35-40

8. Chen, L., Yan, N., Kiang, M., Muth, A.S., Krishna, K.S. Innomotion: a web-based rehabilitation system helping patients recover and gain self-awareness of their body away from the clinic. 2014. In: CHI EA '14 CHI'14 Extended Abstracts on Human Factors in Computing Systems, 233-238

9. Crabtree, A., Hemmings, T., Rodden, T., Cheverst, K., Clarke, K., Dewsbury, G., Hughes, J., Rouncefield, M. 2003. Designing with care: Adapting cultural probes to inform design in sensitive settings. Ergonomics Society of Australia 4, 13

10. Craig, P. MacIntyre, S., Michie, S., Nazareth, N. 2008. Developing and Evaluating Complex Interventions: The New Medical Research Council Guidance. British Medical Journal 33, 5
11. Dahl, Y., Linander, H., Hanssen, G.K. Co-designing interactive tabletop solutions for active patient involvement in audiological consultations. 2014. In: NordiCHI '14 Proceedings of the 8th Nordic Conference on Human-Computer Interaction: Fun, Fast, Foundational, 207-216

12. Dumas, J.S., Redice, J.C. 1999. A Practical Guide to Usability Testing. Revised Edition. Intellect Ltd. 404p.

13. Gale, N.K., Heath G., Cameron, E., Rashid, S., Redwood, S. 2013. Using the framework method for the analysis of qualitative data in multi-disciplinary health research. BMC Medical Research Methodology 13,117

14. Garcia, J.A., Pisan, Y., Tan, C.T., Navarro, K.F. Step kinnection: a hybrid clinical test for fall risk assessment in older adults. 2014. In: CHI EA' 14 CHI '14 Extended Abstracts on Human Factors in Computing Systems 471-474

15. Gregory, J. 2012. How can we assess pain in people who have difficulty communicating? A practice development project identifying a pain assessment tool for acute care. International Practice Development Project 2, 2 Article 6

16. Heslop, P., Blair, P., Fleming, P., Hoghton, M., Marriott, A., Russ, L. 2013. Confidential Inquiry into premature deaths of people with learning disabilities. Norah Fry Research Centre, Bristol. 129p.

17. Howells, G. 1995. Primary Medical Care for People with Learning Disabilities: Overcoming the barriers. Paper presented at Enabling People with a Learning Disability to Use the Health Service. Conference held at St George's Hospital Medical School

18. Kane, S.K., Jayant, C., Wobbrock, J.O., Ladner, R.E. 2009. Freedom to Roam: A Study of Mobile Device Adoption and Accessibility for People with Visual and Motor Disabilities. In: Assets '09 Proceedings of the 11th international ACM SIGACCESS conference on Computers and accessibility, 115-122

19. Krahn, G.L., Hammond, L., Turner, A. 2006. A Cascade of Disparities: Health and Health Care Access for People with Intellectual Disabilities. Developmental Disabilities Research Reviews, 12, 70-82

20. Lancaster University, Institute for Health Research. 2004. Key Highlights of Research Evidence on the Health of People with Learning Disabilities. Lancaster University. $12 \mathrm{p}$

21. Lauer, E., McCallion, P. 2015. Mortality of People with Intellectual and Developmental Disabilities from Select US State Disability Service Systems and Medical Claims Data. Journal of Applied Research in Intellectual Disabilities 28, 394-405

22. Learning Disabilities Observatory. 2015. People with learning disabilities in England 2015: Main report. Public Health England. 97p. 
23. Lennox, N.G., Diggens, J.N., Ugoni, A.M.: 1997. The General Practice Care of People With Intellectual Disability: Barriers and Solutions. Journal of Intellectual Disability Research 41, 5, 380-390

24. Lewis, C., Sullivan, J., Hoehl. 2009. Mobile Technology for People with Cognitive Disabilities and their Caregivers - HCI Issues. UAHCI' 09 Proceedings of the 5th International Conference on Universal Access in Human-Computer Interaction, 385-394

25. Light, J., McNaughton, D. 2013. Putting People First: Re-Thinking the Role of Technology in Augmentative and Alternative Communication Intervention. Augmentative and Alternative Communication 29, 4, 299-309.

26. Medhi, I., Sagar, A., Toyama, K. 2007. Text-free user interfaces for illiterate and semiliterate users. Journal of Information Technologies and International Development 4, 1, 37-50

27. Murphy, J. 2006. Perceptions of Communication between People with Communication Disability and General Practice Staff. Health Expectations 9, 1, 49-59

28. Murphy, J., Cameron, L. 2000. Making Choices at the Time of Transition for Young People with a Learning Disability. University of Stirling.
29. Niemeijer, D., Donnellan, A.M., Robledo, J. 2012. Taking the Pulse of Augmentative and Alternative Communication on iOS. AssistiveWare.

30. Nocon, A. 2006. Equal Treatment - Closing the Gap, Executive Summary. Disability Rights Commission. $122 \mathrm{p}$

31. Norman, D., Euchner, J. 2016. Design for Use: An Interview with Don Norman. Research-Technology Management 58, 1, 15-20.

32. Rogers, S.J., Dawson, G., Vismara, L.A. 2012. An Early Start for Your Child with Autism: Using Everyday Activities to Help Kids Connect, Communicate, and Learn. New York: The Guilford Press, 342p.

33. Simpson, N.J. 1995. Bridging Primary and Secondary Care for People with Learning Disabilities. Advances in Psychiatric Treatment 1, 7, 207-13.

34. World Health Organization. 2018. Definition: intellectual disability http://www.euro.who.int/en/healthtopics/noncommunicable-diseases/mentalhealth/news/news/2010/15/childrens-right-to-familylife/definition-intellectual-disability Archived at: http://www.webcitation.org/6zPF13Wkz 